\title{
METODOLOGÍA DE INVESTIGACIÓN DE LA OFERTA ACADÉMICA EN CARRERAS DE CIENCIAS DEL MAR EN CHILE
}

\author{
Cecilia CANCINO ARAYA ${ }^{1}$ \\ Christian MUNOZ ${ }^{2}$ \\ Sabrina LAMPAS ROSENTHAL ${ }^{3}$
}

RESUMEN: El enfoque de una formación basada en competencias permite la adaptación de las personas al contexto laboral, sin embargo para su implementación se requiere de modificaciones curriculares donde el perfil de egreso sea estructurador y organizador de las competencias a lo largo del curriculum y esté en concordancia con el perfil profesional. La Sociedad Chilena de Ciencias del Mar, en coherencia con uno de sus propósitos relacionados con la formación de capital humano, ha desarrollado una investigación para conocer el estado actual de la oferta académica de las carreras de ciencias del mar, mediante la metodología de análisis multivariados, que permite establecer grados de similitud entre componentes y cuya expresión gráfica es un dendrograma. Esta metodología ha dejado en evidencia una alta sobreposición en los campos laborales, perfiles de egreso, perfiles profesionales y objetivos de la carrera entre carreras distintas, haciendo muy difícil la distinción de ellas, motivo por el cuál actualmente se está trabajando en el levantamiento del perfil profesional, que posibilite la estructuración curricular basada en competencias y de esa forma dar respuesta a las demandas actuales de la sociedad.

PALABRAS ClAVE: Competencias. Curriculum. Análisis multivariado. Ciencias del mar.

\section{Introducción}

La Sociedad Chilena de Ciencias del Mar es una corporación de derecho privado con personalidad jurídica $\mathrm{N}^{\circ} 1404$ de 1994, cuya misión es promover el desarrollo del conocimiento científico, social y cultural relacionado con el ambiente marino en Chile a través de la investigación científica y tecnológica, con el propósito de impulsar políticas sobre su conservación y manejo sustentable.

\footnotetext{
${ }^{1}$ Aluna do Programa de Doctorado en Planificación e Innovación Educativa, da Universidad Alcalá de Henares, com sede no Chile. Membro da Sociedad Chilena de Ciencias del Mar. ceciliacancino.araya@gmail.com

2 Aluno do Programa de Doctorado Sistemática y Biodiversidad, da Universidad de Concepción. christianmz@gmail.com

${ }^{3}$ Professora da Universidad de Valparaíso e da Universidad de las Américas. Aluna do Programa Doctorado Planificación e Innovación Educativa, da Universidad de Alcalá de Henares, com sede no Chile. slampas@yahoo.com.
} 
Desde el año 2008 la Sociedad ha realizado distintas actividades académicas con el propósito de conocer el estado de la formación de capital humano en ciencias del mar en Chile, por lo que hemos realizado un diagnóstico a nivel nacional para conocer acerca de la implementación del cambio hacia la formación basada en competencias en las carreras de ciencias del mar.

En el caso de América Latina, el Proyecto Tuning (2003) resalta la necesidad de que la formación esté basada en competencias, lo que conlleva a una reforma curricular universitaria que incluye modificaciones o cambios curriculares (FLORES; GRAY, 2002), que a su vez modifican el qué y cómo se enseña (TEJADA, 2005). Este enfoque de una formación basada en competencias posibilita un avance porque enfatiza en la globalidad de las capacidades de los individuos y reconstruye los contenidos de la formación con una lógica más productiva, de modo que la utilidad de las competencias radica en que permite que la persona se adapte al contexto laboral (TEJADA, 2005). En este sentido, las competencias identificadas en los curriculos son fundamentales para definir la pertinencia de los programas formativos (VARGAS, 2004), entendiendo la pertinencia como la capacidad de las Instituciones de Educación Superior de responder a las demandas del entorno (UNESCO, 2006). Por otra parte, permite incorporar en la educación un análisis de contenidos e itinerarios de aprendizaje, entre otros.

Para concretar lo anterior en un proyecto formativo, es prioridad la validación previa de los planes de estudio, basándose en un proceso de determinación de las competencias que definen el estándar de desempeño propio de cada profesión u oficio, en un contexto de trabajo (CHILE, 2008). Chile no ha estado ajeno a todo este proceso y son Hawes e Troncoso (2006) los que resaltan la importancia del perfil de egreso como estructurador y organizador de las competencias a lo largo del curriculum, por lo tanto es el que articula con el contexto laboral. Esto implica que la malla curricular debe ser coherente con el perfil de egreso y éste, a su vez, debe ser pertinente al perfil profesional.

En los últimos años se ha invertido altas sumas de dinero en pro de mejorar la cobertura y calidad de la Educación en Chile (CHILE, 2008), toda vez que las modificaciones en el mundo del trabajo han generado nuevas necesidades en los procesos de formación (TEJADA, 2005), lo que determina la periodicidad en la revisión de los perfiles de egreso.

Sin embargo, nuestra percepción es que los procesos de rediseño curricular en ciencias del mar han sido realizados de manera individual por las distintas unidades académicas lo que dificulta la movilidad estudiantil. Es por esto que la Sociedad Chilena 
de Ciencias del Mar ha realizado un diagnóstico a nivel nacional de las ofertas académicas para conocer el grado de similitud de los programas de pregrado, por lo tanto el objetivo del presente trabajo es dar a conocer la utilización de metodologías de análisis multivariado que permiten la identificación de niveles de similitud entre componentes para la creación los dendrogramas, con el fin de conocer el estado de la formación de pregrado de las carreras de ciencias del mar.

\section{Metodología}

En el año 2009 se realiza una revisión de todas las Instituciones de Educación Superior de Chile, que encontrándose en la web impartan programas de pregrado y postgrado en el área de ciencias del mar. Para ello se utilizó como criterio de selección que el programa declarara estudios relacionados con organismos que habitan en el mar. Una vez seleccionados, se envía una carta de presentación de la investigación a cada Decano, Director y/o Jefe de Carrera para verificar la selección y su aprobación de participación.

La información disponible en las páginas web institucionales, presentada de forma heterogénea, corresponde a perfiles profesionales, perfiles de egreso, descripciones de las carreras, objetivos y campos laborales. Las mallas curriculares se encuentran disponibles para todas las carreras.

En el año 2010 se da inicio a la selección de datos, que corresponden a las palabras que forman parte de las declaraciones institucionales y que están relacionadas con el proceso de formación bajo todos los aspectos posibles. Con esta base de datos se construye las matrices para el análisis, que consideran la presencia de la palabra con categoría 1 y la ausencia con categoría 0 , generando así matrices binarias de similitud.

\section{Título: Matrices para el análisis}




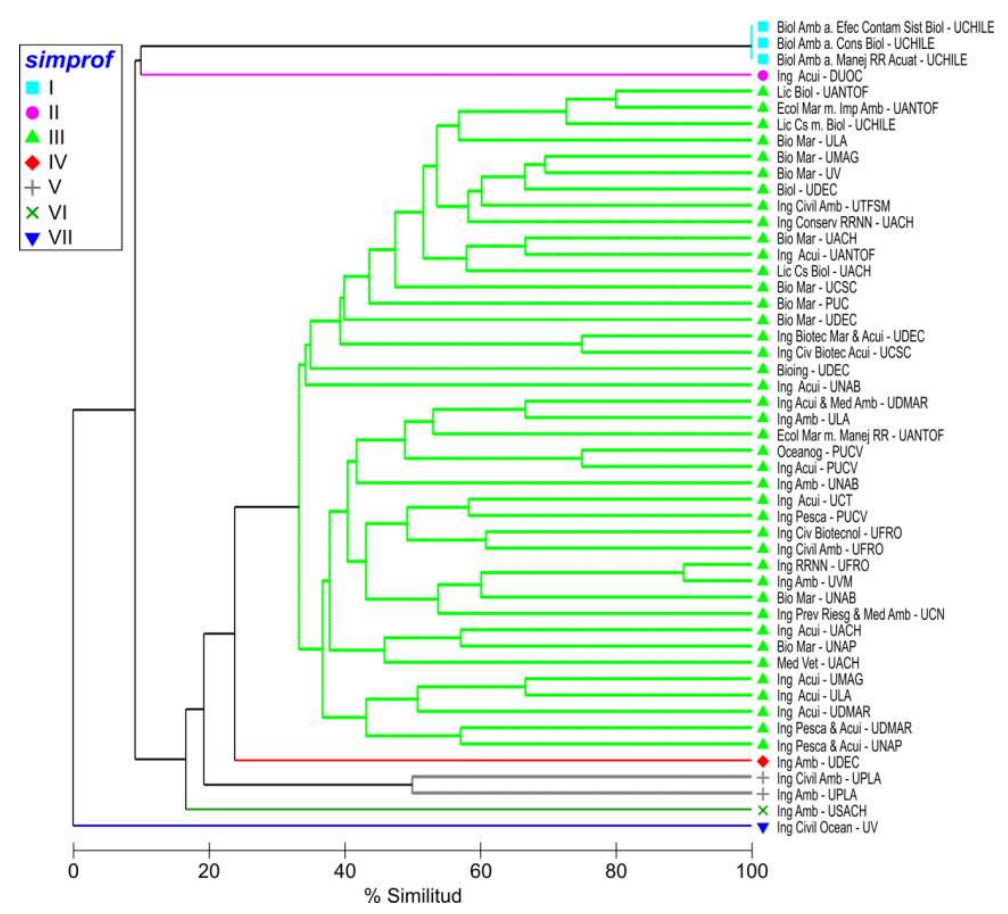

Fuente: Elaboración propia.

Los instrumentos estadísticos seleccionados para el análisis de datos corresponden al programa Primer v6.1.2. (CLARKE; GORLEY, 2006), que se utiliza ampliamente en análisis multivariados, ya que permite establecer grados de similitud entre componentes y cuya expresión gráfica es un dendrograma. Los componentes analizados son las carreras de pregrado relacionadas con Ciencias del Mar y las variables que permiten agrupar por similitud son las palabras clave seleccionadas de las descripciones de la carrera, perfiles de egreso, perfiles profesionales, mallas curriculares, campos laborales y objetivos de las carreras. Los dendrogramas se construyen utilizando el algoritmo de la media aritmética por pares no ponderados UPGMA (SNEATH; SOKAL, 1973) sobre matrices de similitud, construidas con el índice de Sørensen (1948), basados en los registros binarios de presencia/ausencia de las variables antes mencionadas. Además, para establecer grupos estadísticamente significativos $(\mathrm{p}<0.05)$, se realiza un perfil de similitud SIMPROF (CLARKE; GORLEY, 2006; CLARKE; SOMERFIELD; GORLEY, 2008), utilizando 50.000 iteraciones. Gráficamente, cada grupo significativo que se forma es representado por un color y un símbolo característico, que se ubica en el cuadro denominado SIMPROF.

Finalmente, se realiza un análisis de porcentaje de similitud SIMPER (CLARKE, 1993) para identificar las variables que explican en al menos un 70\% la estructuración de los datos. 


\section{Resultados}

Se analizaron las carreras de ciencias del mar que se imparten en Chile, sin hacer distinción del tipo de Institución del sistema de Educación Superior de Chile. En cada análisis se observa un número distinto de datos, ya que las carreras declaran de manera diferencial la información de sus ofertas académicas.

\section{1.- Dendrograma de campos laborales}

\begin{tabular}{|c|c|c|}
\hline \multirow[t]{2}{*}{ Sirrilitud promedio (ja) } & Grupo I $[22,45]$ & Grupo II $(20,64)$ \\
\hline & \multicolumn{2}{|c|}{ Contribución ["sa) } \\
\hline Acuicultura & & 17.19 \\
\hline Conservación & 6.82 & \\
\hline Cultivos & & 21.02 \\
\hline L esamollo Sustentatle & 6.33 & \\
\hline E esarrollo ecnológico & 7.30 & \\
\hline Empresa piblica & 3.6 & \\
\hline E valuación & 6.22 & \\
\hline Gestión & 6.60 & \\
\hline In formación C ientífica & 4.28 & \\
\hline Investigación & 4.17 & \\
\hline Liderazgo & & 13.97 \\
\hline Lledio Ambiente & 3.70 & \\
\hline |laltidis ciplinariedad & 4.06 & \\
\hline Productividad & & 13.53 \\
\hline Proyedos & 6.82 & \\
\hline Soluciön Pmblemas & 11.37 & 7.93 \\
\hline Total contribución & 71.37 & 73.63 \\
\hline Núnero de iterrg & 12 & 5 \\
\hline
\end{tabular}

Fuente: Elaboración propia.

El perfil de similitud muestra la presencia de 7 grupos estadísticamente significativos, entre los cuales destaca el grupo III que anida a la mayor cantidad de carreras de ciencias del mar de Chile, por lo que según esta variable es difícil distinguir entre carreras sobre la base de los campos laborales que declaran. El grupo I presenta $100 \%$ de similitud del campo laboral declarado para tres carreras de biología ambiental.

\section{2.- Dendrograma perfil profesional}




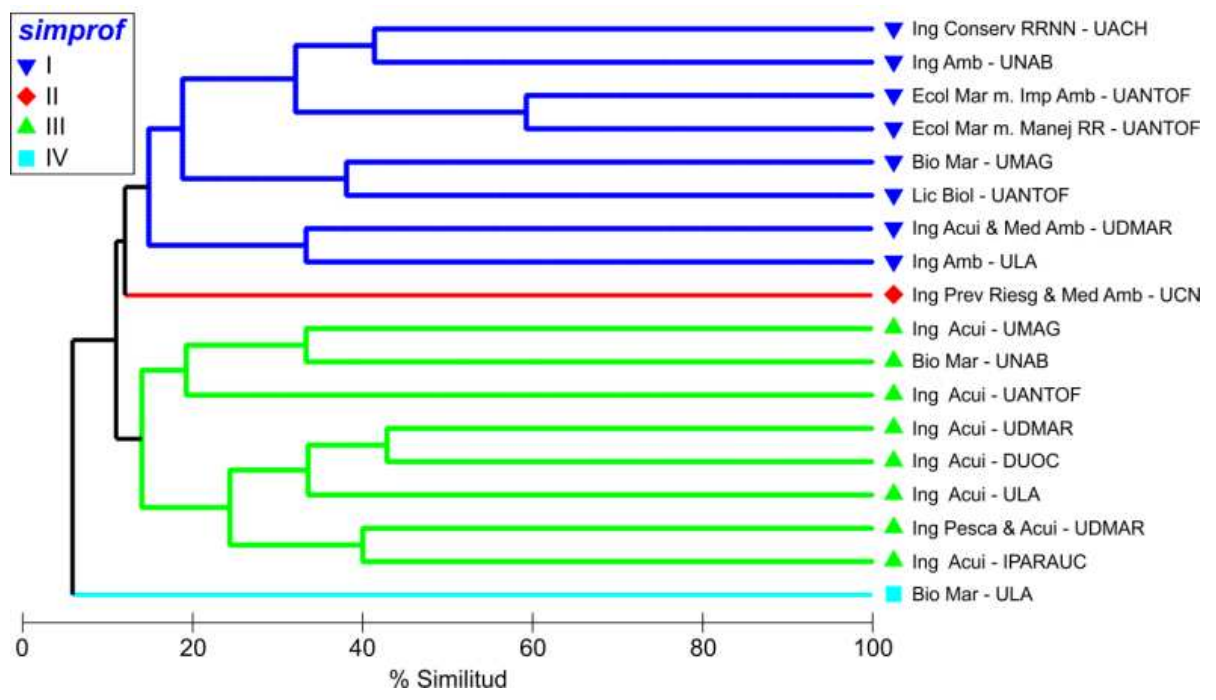

\begin{tabular}{|c|c|c|c|}
\hline \multirow[t]{2}{*}{ SImllitud promodlo (\%) } & Grupo I (100.00) & Grupo III (38.01) & Grupo V $(\mathbf{6 0 . 0 0 )}$ \\
\hline & \multicolumn{3}{|c|}{ Contribución (\%) } \\
\hline Asesorías & 7.69 & & 20.00 \\
\hline Biología & 7.69 & & \\
\hline Conservación & 7.69 & & \\
\hline Consultoras & & 10.53 & 20.00 \\
\hline Explotación & & & 20.00 \\
\hline Fisica & 7.69 & & \\
\hline Innovación & 7.69 & & \\
\hline Instituciones privadas & & 25.48 & \\
\hline Instituciones públicas & & 23.77 & \\
\hline Investigación & & 6.28 & \\
\hline Matemática & 7.69 & & \\
\hline Medio Ambiente & 7.69 & & \\
\hline Producción comercial & 7.69 & & \\
\hline Química & 7.69 & & \\
\hline Recursos Naturales & & & 20.00 \\
\hline Tecnología & 7.69 & & \\
\hline Universidad & & 8.86 & \\
\hline Total contrlbuclón & 76.92 & 74.92 & 80.00 \\
\hline Número de items & 10 & 6 & $\xi$ \\
\hline
\end{tabular}

Fuente: Elaboración propia.

El perfil de similitud logra diferenciar la presencia de 4 grupos significativos; de estos, se destaca la formación de un grupo conformado principalmente por carreras con orientación a la ecología y conservación (grupo I) mientras que el grupo III muestra la agrupación de carreras enfocadas hacia la acuicultura.

\section{3.- Dendrograma perfil de egreso}

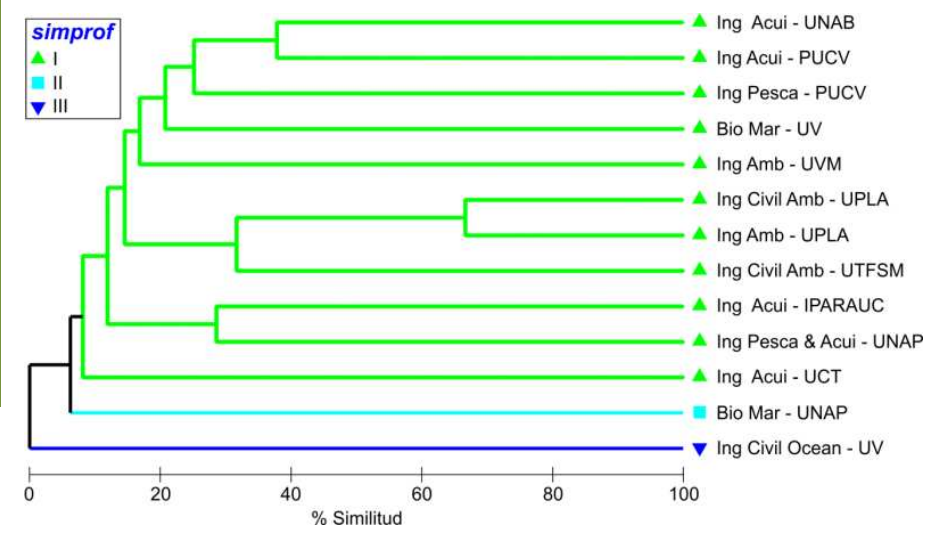

\begin{tabular}{lr}
\hline Similitud promedio (\%) & \multicolumn{1}{c}{ Grupo I (15.75) } \\
\cline { 2 - 2 } & Contribución (\%) \\
\hline Acuicultura & 8.23 \\
Cultivos & 6.02 \\
Gestión & 10.23 \\
Ingeniería & 6.66 \\
Medio ambiente & 10.63 \\
Producción & 9.83 \\
Proyectos & 5.83 \\
Solución de problemas & 5.49 \\
Tecnología & 9.83 \\
& \\
Total contribución & $\mathbf{7 2 . 7 4}$ \\
Número de items & $\mathbf{9}$ \\
\hline
\end{tabular}




\begin{tabular}{lrr}
\hline Similitud promedio (\%) & \multicolumn{2}{c}{ Grupo I (19.27) } \\
\cline { 2 - 3 } & \multicolumn{2}{c}{ Contribución (\%) } \\
\hline Administrar & & 11.39 \\
Investigación & & 7.24 \\
Ciencias de la Ingeniería & 13.84 & \\
Conocimiento científico & & 17.48 \\
Gestión & 33.33 & 23.49 \\
Tecnología & & 14.86 \\
Trabajo de equipo & 33.33 & \\
& & \\
Total contribución & $\mathbf{8 0 . 5 1}$ & $\mathbf{7 4 . 4 7}$ \\
Número de items & $\mathbf{3}$ & $\mathbf{5}$ \\
\hline
\end{tabular}

Fuente: Elaboración propia.

El perfil de similitud determina la existencia de 3 grupos significativos, siendo el grupo I el que anida al mayor porcentaje de las carreras, con 9 palabras explican el $72,4 \%$ del clado.

\section{4.- Dendograma objetivos de carrera}

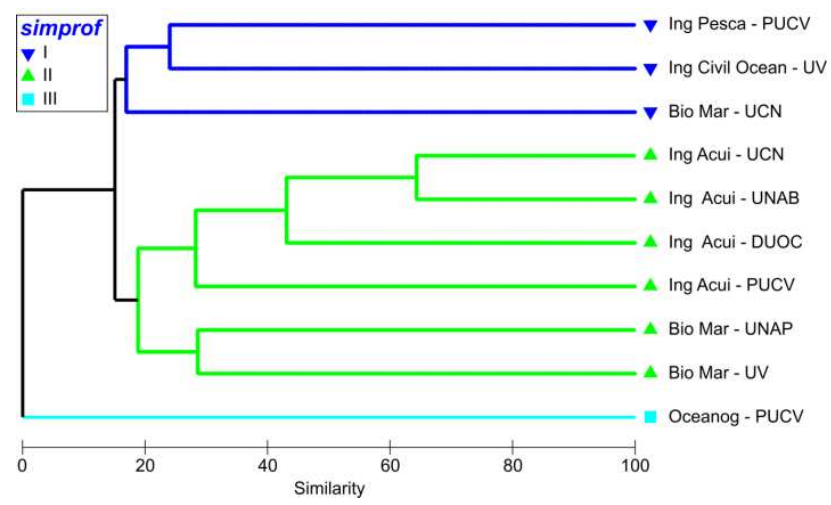

Fuente: Elaboración propia.

El perfil de similitud determinó la existencia de tres grupos significativos. Los grupos I y III se diferencian estadísticamente, sin embargo ambos están formados por carreras de Ingeniería y Biología Marina. La tabla Simper muestra el porcentaje de contribución de cada palabra a la conformación de los grupos internos.

\section{Dendrograma mallas curriculares}




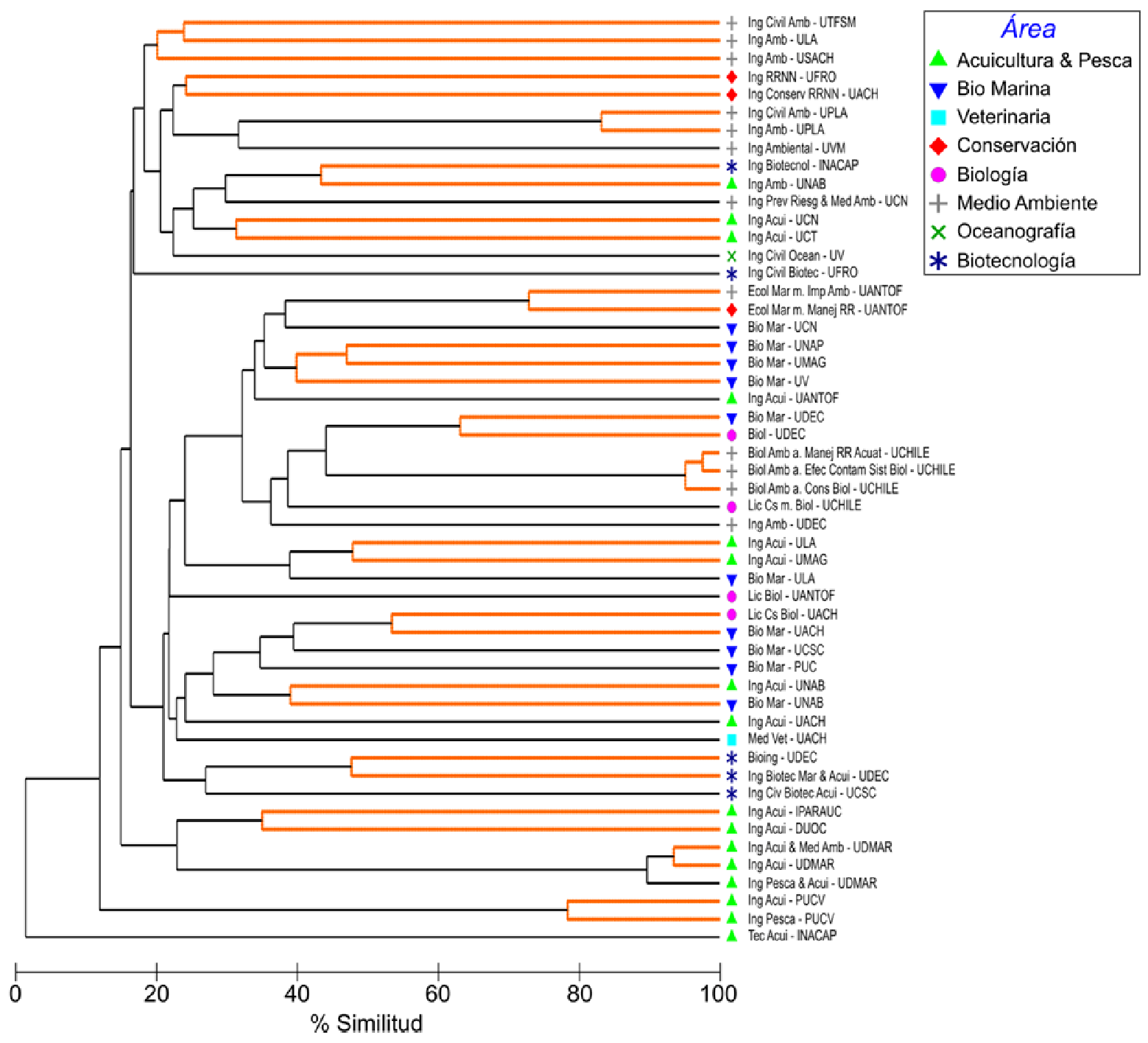

Fuente: Elaboración propia.

Para el análisis de las mallas curriculares, se asignó una figura y un color a las carreras según el área característica a la cual pertenece la carrera. Los resultados muestran que los cluster se conforman por carreras diferentes, lo que en parte puede ser explicado por los nombres de las asignaturas y no necesariamente por sus contenidos. Las asociaciones de carreras por mallas curriculares estarían confirmando la sobreposición entre distintas carreras, como se ha evidenciado con las variables anteriores. Por otro lado, similitudes cercanas al $80 \%$ se observan en aquellas carreras que siendo distintas, pertenecen a la misma institución.

Al analizar que sucede con las carreras por área en relación a la variable malla curricular observamos los siguientes dendrogramas: 


\section{A.- Dendrograma Biología Marina:}

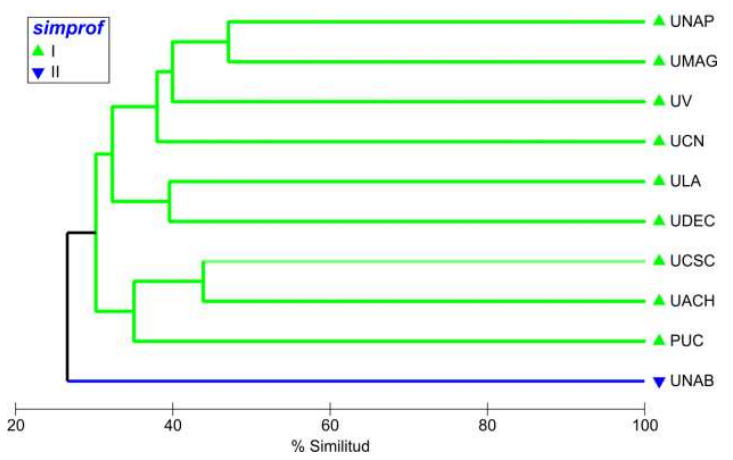

Fuente: Elaboración propia.

\section{B.- Dendrograma Ingeniería enAcuicultura}

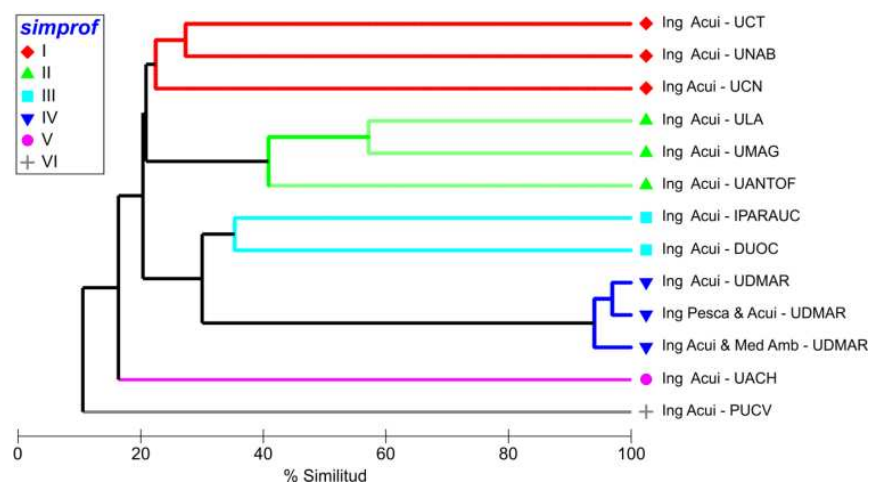

Fuente: Elaboración propia.

\section{C.- Dendrograma Ingeniería Pesquera}

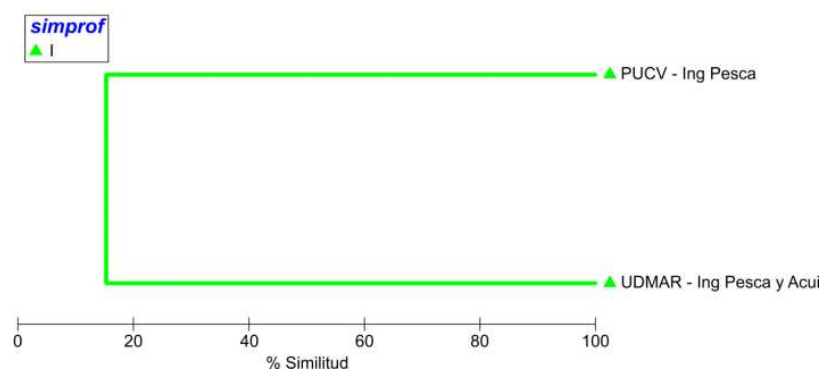

Fuente: Elaboración propia. 


\section{D.- Dendrograma Ingeniería Ambiental}

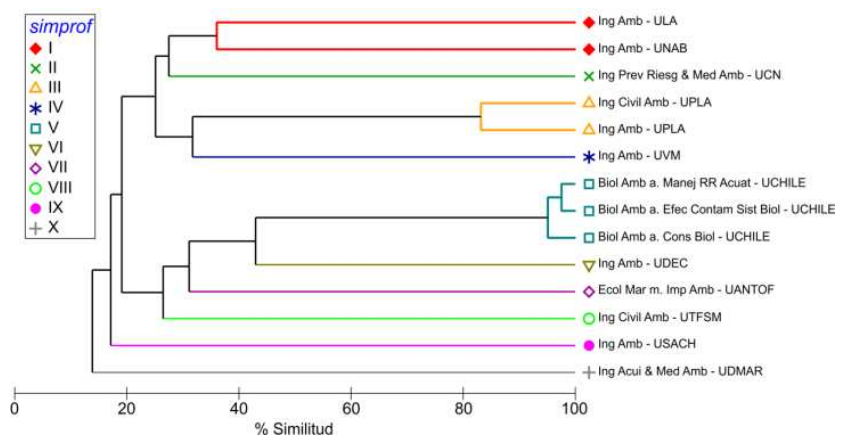

Fuente: Elaboración propia.

\section{E.- Dendrograma Biología y Licenciatura}

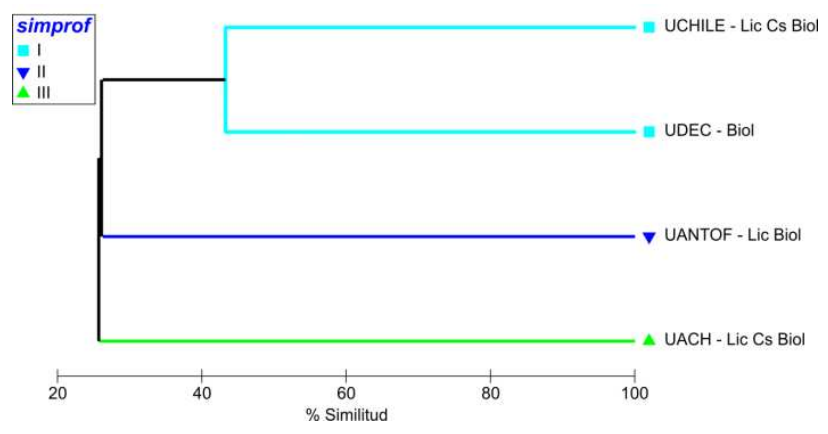

Fuente: Elaboración propia.

\section{F.- Dendrograma Biotecnología}

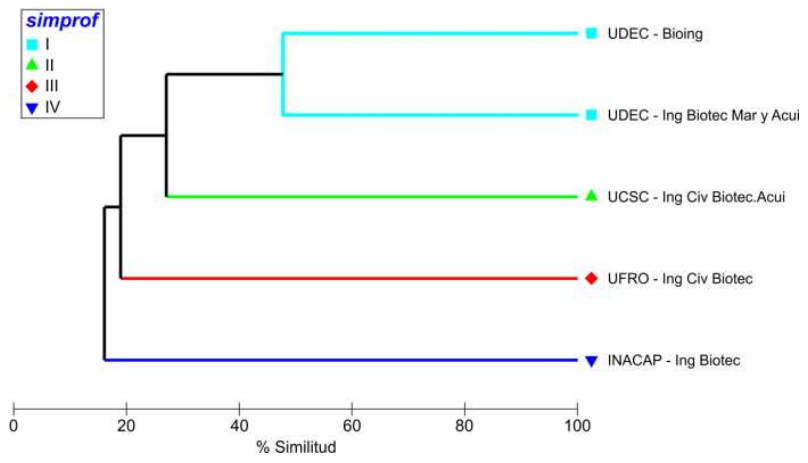

Fuente: Elaboración propia. 


\section{G.- Dendrograma Recursos Naturales}

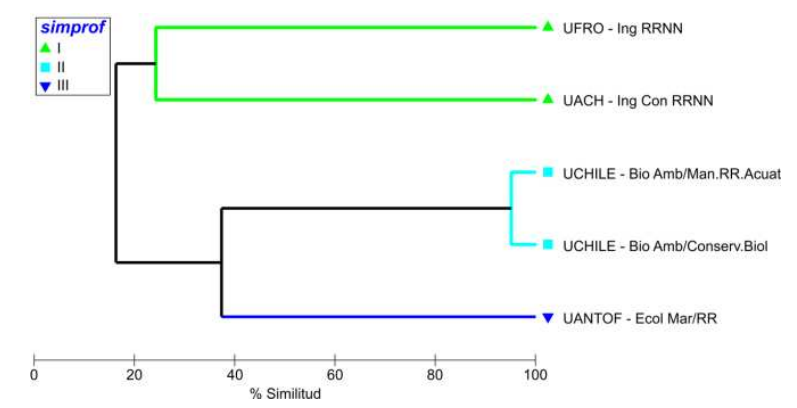

Fuente: Elaboración propia.

\section{Discusiones}

La mayoría de las Universidades chilenas declaran tener un curriculum basado en competencias, incorporando las competencias tanto al lenguaje como a los contenidos universitarios (YÁNIZ, 2008), sin embargo la coherencia que existe entre los objetivos del sistema educativo, los procesos y los resultados sigue estando bajo los niveles deseados (MARTÍNEZ-MEDIANO, 1996), lo que en el caso de las carreras de ciencias del mar pudiera ser explicado según lo demuestran estos resultados, por la falta de distinción de los campos ocupacionales de las profesiones y la diversidad de perfiles profesionales declarados por las instituciones lo que a su vez explicaría las diferencias de los perfiles de egreso y objetivos para iguales carreras profesionales. Del mismo modo podemos explicar las bajas similitudes en las mallas curriculares, aunque es preciso hacer una evaluación de los programas de las asignaturas, ya que las diferencias pudieran ser debido a los nombres que se utilizan para estructurar dichas mallas.

Las Universidades han invertido mucho en los procesos de rediseño curricular, sin embargo llama la atención que estando bajo la lógica de las competencias, las carreras declaren indistintamente el mismo tipo de información con denominaciones diferentes, como es el caso de los perfiles profesionales y de egreso.

La Sociedad Chilena de Ciencias del Mar ha realizado talleres ampliados en los últimos dos Congresos de Ciencias del Mar, dando a conocer estos resultados en talleres específicos con los jefes de carrera de biología marina y de acuicultura de todas las carreras que a la fecha están vigentes, a partir de los cuales se han logrado diferenciar los campos laborales y lo que la academia considera que debiesen ser los perfiles profesionales de cada una de ellas. En estos momentos se encuentra en etapa de implementación de una encuesta a nivel nacional para los empleadores, a partir de la cual, 
en conjunto con la información anterior, se levantarán los correspondientes perfiles profesionales y se analizará la articulación con los programas de postgrado en el área de las ciencias del mar.

\section{RESEARCH METHODOLOGY OF ACADEMIC CAREER OFFER OF MARINE SCIENCE IN CHILE}

ABSTRACT: The competence based model allows the students to develop the skills they need in the work context. To introduce this model, curricular adaptations are required, where the academic profile must organized the competences through the curriculum and must be in agreement with the professional profile. One of the purposes of the "Marine Science Chilean Society" is related to human capital formation, so it has develop a research to know the actual state of the academic offer in marine science, using a multivariate analysis that shows the similarity between variables in a dendrogram. The results evidence a high overlap in the fieldwork, academic profiles, professional profiles and career goals between different progammes, so the distinction between them is very hard. Actually the "Marine Science Chilean Society" is working in the construction of the professional profile, to develop competence based degree programmes that respond to society requests.

KEYWORDS: Competences. Curriculum. Multivariate analysis. Marine science.

\section{REFERÊNCIAS}

CHILE. Consejo Asesor Presidencial para la Educación Superior. Los desafíos de la educación superior. Chile: Informe del Consejo Asesor Presidencial, 2008.

CLARKE, K. R. Non-parametric multivariate analyses of change in community structure. Australian Journal of Ecology, [S.1.], n.18, p.117-143, 1993.

CLARKE, K. R.; GORLEY, R. N. PRIMER v6: user manual/tutorial. Plymouth: PRIMER-E Ltd, 2006.

CLARKE, K. R.; SOMERFIELD, P. J.; GORLEY, R. N. Testing of null hypotheses in exploratory community analyses: similarity profiles and biota-environment linkage. Journal of Experimental Marine Biology and Ecology, Plymouth, v.366, p.56-69, 2008.

FLORES, F.; GRAY, J. El espíritu emprendedor y la vida wired: el trabajo en el Ocaso de las Carreras. Relaciones del Trabajo, [S.1.], v.14, n.37, 2002.

HAWES, G.; TRONCOSO, K. Lineamientos para la transformación del curriculum de formación profesional en las Carreras de la Red RINAC. Valdivia: Proyecto Mecesup AUS 402, 2006.

MARTÍNEZ-MEDIANO, C. Investigación evaluativa sobre los factores de eficacia de los centros asociados de la UNED, en Investigar para mejorar la calidad de la 
universidad, UNED. Revista Iberoamericana de Educación Superior a Distancia, Madrid, v.3, n.1, 1996.

PROYECTO TUNING AMÉRICA LATINA. [S.1.], 2003. Disponible en:

$<$ http://tuning.unideusto.org/tuningal>. Acceso en: 15 ago. 2012.

SNEATH, P. H. A.; SOKAL, R. R. Numerical taxonomy. San Francisco: W. H. Freeman, 1973

SØRENSEN, T. A. A method of establishing groups of equal amplitude in plant sociology based on similarity of species content, and its application to analyses of the vegetation on Danish commons. Kongelige Danske Videnskabernes Selskabs Biologiske Skrifter, [S.1.], v.5, p.1-34, 1948.

TEJADA, J. El trabajo por competencias en el prácticum: cómo organizarlo y cómo evaluarlo. Revista Electrónica de Investigación Educativa, Baja California, v.7, n.2, 2005.

UNESCO. Instituto Internacional de la UNESCO para la Educación Superior en América Latina y el Caribe (IESALC). Informe sobre la educación superior en América Latina y el Caribe 2000-2005. La metamorfosis de la educación superior. Venezuela:

Metrópolis, 2006.

VARGAS F. 40 preguntas sobre competencia laboral. Montevideo: Cinterfor, 2004.

YÁNIZ, C. Las competencias en el currículo universitario: implicaciones para diseñar el aprendizaje y para la formación del profesorado. Revista de Docencia Universitaria, Santiago de Compostela, v.6, n.1, p.31-39, 2008. 